\title{
Pre-Operative Endovascular Embolization of a Cerebellar Haemangioblastoma
}

\author{
A Case Report
}

\author{
A. Abo-Al Hassan ${ }^{a}$ M. Ismail ${ }^{b}$ S.M. Panda ${ }^{a}$ \\ Departments of a Neurosurgery and ${ }^{b}$ Radiology, Ibn Sina Hospital, Kuwait
}

\section{Key Words \\ Haemangioblastoma • Cerebral angiography • \\ Neuro-interventional techniques $\cdot$ Polyvinyl alcohol}

\begin{abstract}
Objective: To present an interesting case of pre-operative embolization of a cerebellar haemangioblastoma. Clinical Presentation and Intervention: A 36-year-old male presented with gradual, progressive headache and a positive family history of von Hippel-Lindau syndrome. MRI of the brain revealed a right cerebellar solid mass and cerebral angiography demonstrated its extensive hypervascular nature. The mass was embolized with polyvinyl alcohol prior to surgical resection, which resulted in improvement of the patient's symptoms. Conclusion: Pre-operative embolization of a haemangioblastoma is a useful procedure that can potentially decrease the morbidity and mortality of its surgical resection.

Copyright $\odot 2006$ S. Karger AG, Basel
\end{abstract}

\section{Introduction}

Haemangioblastomas (HBs) are histologically benign tumours that occur exclusively within the neuraxis, most commonly in the posterior fossa. They account for 1.5-
$2.5 \%$ of all intracranial tumours. HBs may occur sporadically as isolated tumours of the cerebellum or may represent a familial disorder as part of the von HippelLindau (VHL) complex [1].

HBs can be difficult to resect owing to its hypervascularity and location in the posterior fossa [2]. High rates of morbidity and mortality are associated with uncontrollable intra-operative haemorrhage [3]. We report a case of pre-operative embolization of a cerebellar HB.

\section{Case Report}

A 36-year-old male presented with several months' history of gradual and progressive headache, mainly in the right occipital region. There was a positive family history of VHL syndrome. On clinical examination, there was a slight nystagmus to the left side. No associated cerebellar symptom, visual disturbance or other neurological symptoms were observed. The patient underwent brain MRI, which revealed a $3 \times 3 \mathrm{~cm}$ right cerebellar solid mass, hyperintense on a $\mathrm{T}_{2}$-weighted image and hypo-intense on a $\mathrm{T}_{1}$ weighted image, with thick irregular peripheral enhancement after intravenous gadolinium injection (fig. 1). Following MRI, the patient underwent diagnostic cerebral angiography, which showed a hypervascular mass in the inferior aspect of the right hemisphere with a large arterial feeder arising from the right anterior inferior cerebellar artery (AICA), and a smaller feeder from the right posterior inferior cerebellar artery (fig. 2). This was followed by pre-operative embolization of the tumour's vascular bed on the day prior to surgery. The embolization proce-

\section{KARGER}

Fax +4161306 1234

E-Mail karger@karger.ch

www.karger.com
(C) 2006 S. Karger AG, Basel

$1011-7571 / 06 / 0156-0459 \$ 23.50 / 0$

Accessible online at:

www.karger.com/mpp
Dr. Ali Abo-Al Hassan

Ibn Sina Hospital, Neurosurgical Department

Mishref, Block 6, Street 3, Jada I, House 11, PO Box 244

Mishref 40153 (Kuwait)

Tel. +965 481 8253, Fax +965 484 9226, E-Mail ali_aboalhassan@hotmail.com 

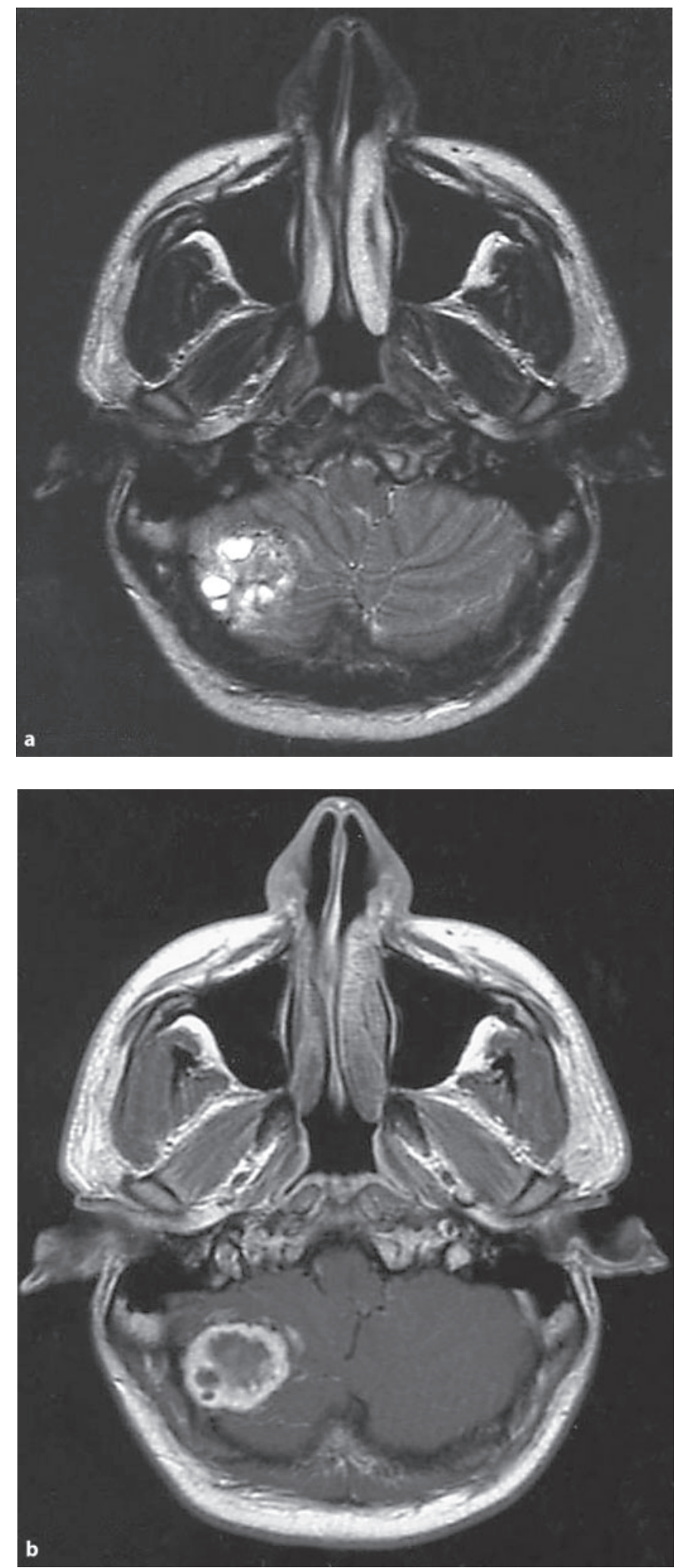

Fig. 1. a Axial $\mathrm{T}_{2}$-weighted image shows a hyperintense right cerebellar mass. b Axial $\mathrm{T}_{1}$-weighted image after gadolinium injection demonstrates thick irregular peripheral enhancement.
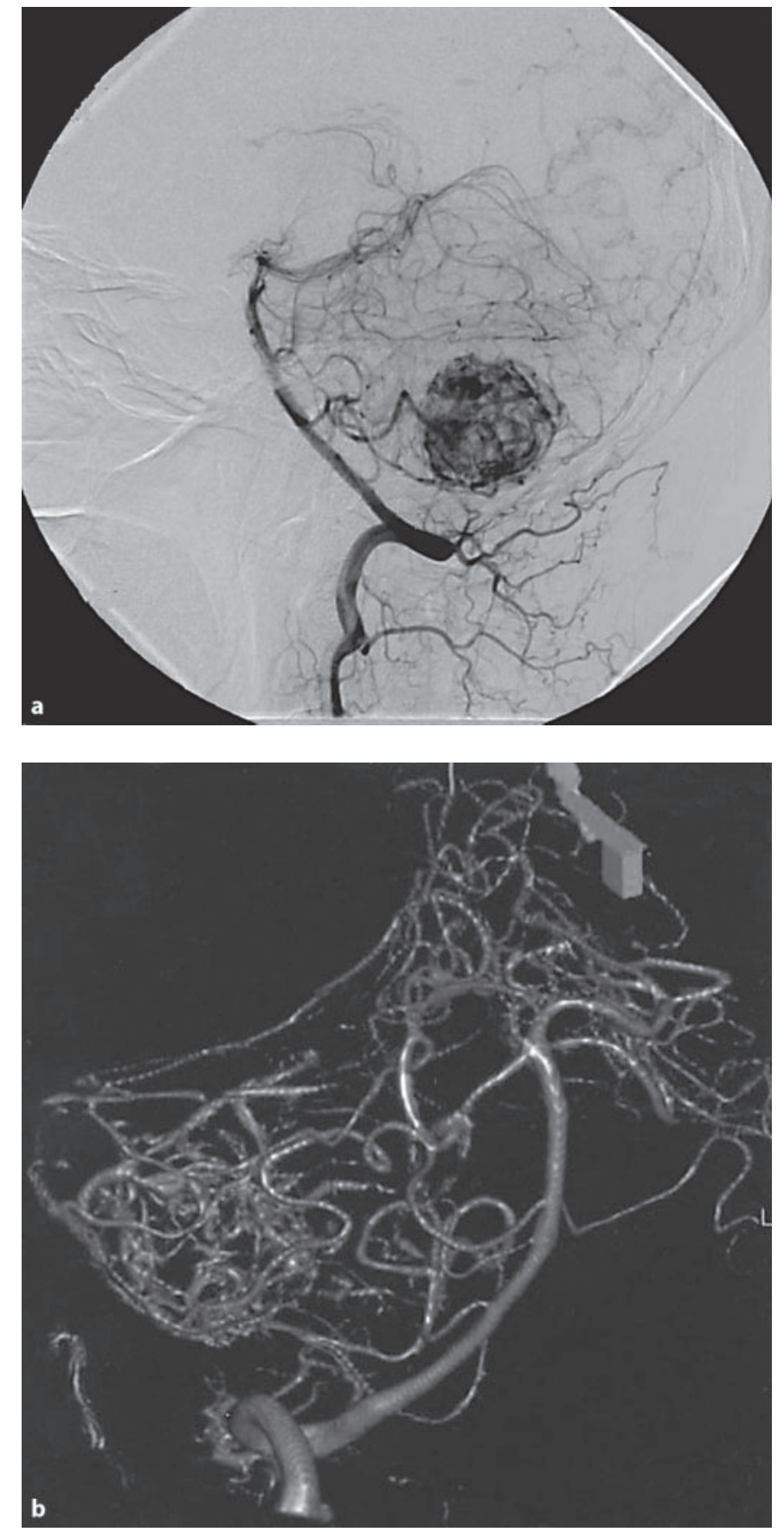

Fig. 2. a Lateral right vertebral angiogram. b Three-dimensional reconstruction, anterior-oblique view, demonstrating a right cerebellar hypervascular mass, with a large arterial feeder from the right AICA. 

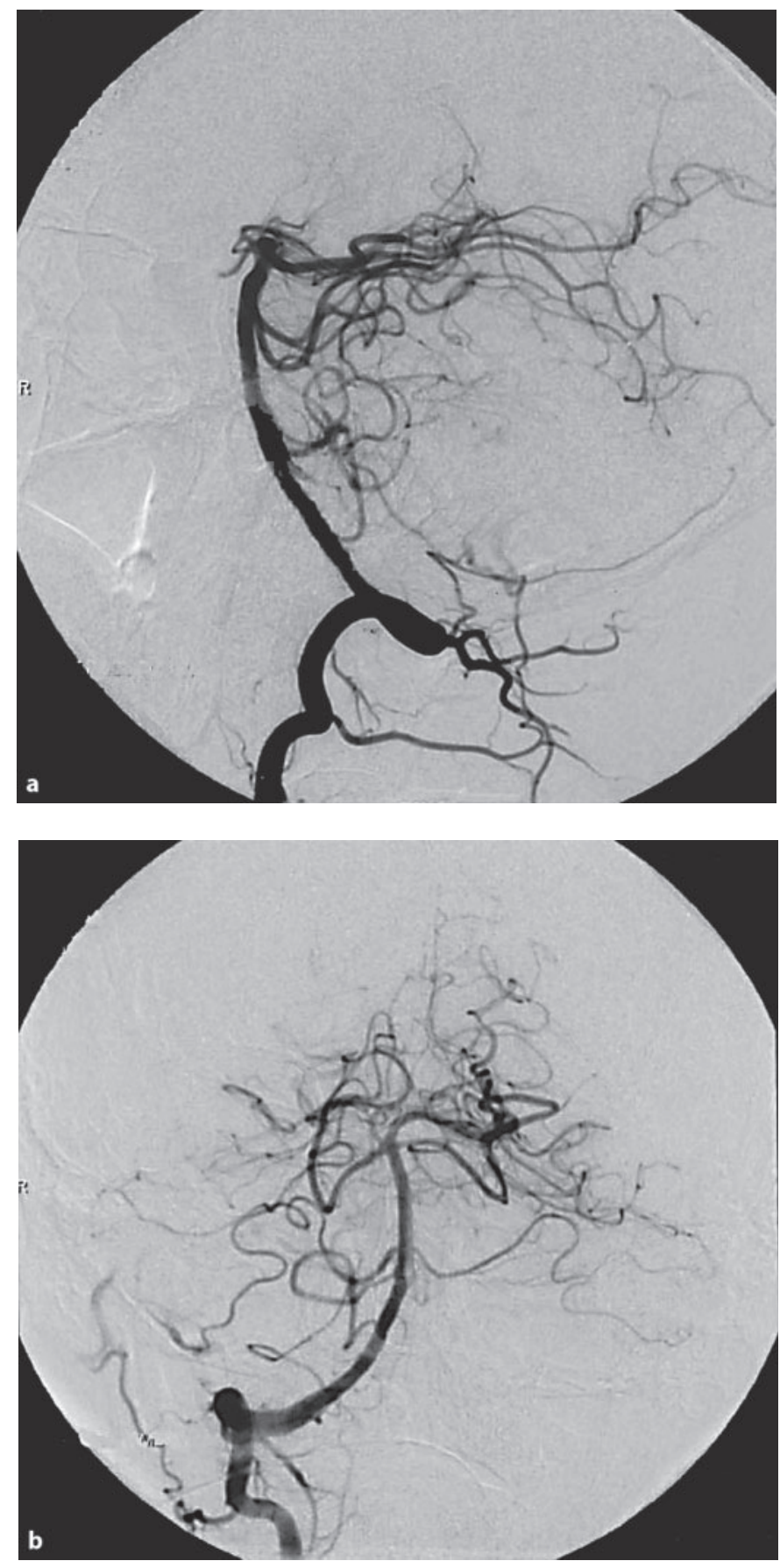

Fig. 3. Lateral (a) and frontal (b) right vertebral artery angiogram after embolization demonstrating almost complete devascularization of the tumour.

dure involved transarterial placement of a 5-french guiding catheter in the right vertebral artery via a right femoral artery sheath. Under road map guidance, a 0.014 -inch microcatheter and microguide wire were navigated distally into the right AICA close to the tumour.

Endovascular Embolization of a Cerebellar Haemangioblastoma
Polyvinyl alcohol (PVA) particles (size 45-150 $\mu \mathrm{m})$ were mixed with non-ionic contrast media and infused manually with a 3-ml syringe under fluoroscopic monitoring through the microcatheter into the distal right AICA. Fluoroscopic monitoring during embolization is mandatory to detect early reflux of PVA, thus preventing reflux into other parts of posterior circulation. The infusion was stopped as soon as a small amount of reflux was noticed at the microcatheter tip. As a result of the procedure, there was complete devascularization of the tumour (fig. 3). The patient underwent mid-right suboccipital retromastoid craniectomy and total excision of the right cerebellar tumour on the following day with minimal bleeding at the surgical site.

\section{Discussion}

HBs are benign, usually highly vascular tumours that may be either solid or cystic, with a higher proportion (70\%) of cerebellar HBs being cystic. In cystic lesions, the solid component is a small nubbin, commonly described as a mural nodule. The cyst contains clear, highly proteinaceous fluid. Because of their location in the posterior fossa and spinal cord, HBs can be difficult to remove [2]. The vascularity of the tumour also contributes to the difficulty encountered during surgical resection.

HBs may occur sporadically as isolated tumours of the cerebellum or may represent a familial disorder as part of the VHL complex. The latter disorder is transmitted as an autosomal dominant trait with varying penetrance. The average age at onset of symptoms in familial cases is 30 years, with a range of 3-83 years. Non-familial or sporadic cases of cerebellar HB tend to present later, with a mean age at presentation of 42 years. The symptoms and signs vary to some extent, depending upon the precise location of the tumour in the posterior fossa. Headache is the most common symptom, being present in $95 \%$ of cases.

Vomiting is the next most common symptom, and may be due to obstructive hydrocephalus or to irritation of the vagal nucleus from the tumour. Vertigo, gait disturbance, diplopia and ataxia are other symptoms of cerebellar HB. Most HBs occur in the posterior fossa, clustered around the fourth ventricle [4]; less commonly, they are found in the supratentorial compartment [5] or in the spinal cord [6]. Retinal HBs occur in $6 \%$ of the patients with cerebellar HBs [7].

Care is needed during PVA particle embolization to prevent reflux into other parts of the circulation. This could be achieved by positioning the microcatheter as close as possible to the tumour, and by slow hand injection of the PVA/contrast mixture under continuous fluoroscopic monitoring to detect any decrease in antegrade flow or early reflux. 
Modern neuro-interventional techniques, including the use of microcatheters, microguide wires and highresolution angiography with 3-dimensional reconstruction and road-mapping techniques [8], have enabled devascularization of vascular intracranial lesions prior to surgery [6], and surgical removal of these lesions is facilitated by embolization. PVA is our preferred embolic agent, as these particles quickly devascularize the nidus by penetrating deep into the microcirculation; moreover, only a small amount $(0.25-2.0 \mathrm{ml})$ is required for angiographic devascularization of most HBs.

Baumgartner et al. reported intratumoural haemorrhage complicating PVA embolization in 1 out of 10 cases [9]. Joseph et al. reported raised intracranial pressure after embolization, which necessitated emergency craniotomy with ventriculostomy in 1 out of 9 cases [9]. All patients undergoing embolization must be observed closely and need intravenous steroid because of the danger of tumour swelling [9]. Ideally, resection is done 48$72 \mathrm{~h}$ after embolization, as the effect of PVA is temporary.

\section{Conclusion}

Pre-operative embolization of HBs is a useful procedure that can potentially decrease the morbidity and mortality of surgical resection of HBs. These tumours usually require a small amount of PVA particles. Surgery is generally performed within $72 \mathrm{~h}$ and careful observation of the patients in the hospital is required in case of complications from tumour swelling or haemorrhage.

\section{References}

1 Bonghey AM, Fletcher NA, Harding AE: Central nervous system hemangioblastoma: a clinical and genetic study of 52 cases. J Neurol Neurosurg Psychiatry 1990;54:644-648.

2 Palmer DG: Hemangioblastomas: a review of 81 cases. Acta Neurochir 1972;27:125148.

3 Djindjian M: Successful removal of a brain stem hemangioblastoma. Surg Neurol 1986; 25:97-100.
4 Lee SR, Sanches J, Mark AS, Dillon WP, Norman D, Newton TH: Posterior fossa hemangioblastomas: MR imaging. Radiology 1989; 171:463-468.

5 Richmond BK, Schmidt JH 3rd: Congenital cystic supratentorial hemangioblastoma. Case report. J Neurosurg 1995;82:113-115.

6 Murota T, Symon L: Surgical management of hemangioblastoma of the spinal cord: a report of 18 cases. Neurosurgery 1989;25:699708 .
7 Moore AT, Maher ER, Rosen P, Gregor Z, Bird AC: Ophthalmological screening for von Hippel-Lindau disease. Eye 1991;5:723728.

8 Eskridge JM: Interventional radiology. Radiology 1989;172:991-1006.

9 Eskridge JM, McAuliffe W, Harris B, Kim DK, Scott J, Winn HR: Preoperative endovascular embolization of craniospinal hemangioblastomas. Am J Neuroradiol 1996; 17:525-531. 